\title{
DIPTERA OF LABRADOR
}

\author{
By Charles W. Johnson
}

Boston Society of Natural History

This list was for the most part prepared some time ago, but was laid aside to await the determination of few species. In the meantime, however, other work intervened. The list represents the results of a number of expeditions by zoölogists and collectors including Alpheus S. Packard in 1860 and 1864. Samuel Henshaw in 1881, Lucien M. Turner in 1882 and 1883, J. D. Sornborger in 1892 and 1897, A Stecker and J. D. Sornborger in 1899, Dr. C. W. Townsend and G. M. Allen 1906, Owen Bryant 1908 and O. L. Austin, Jr., 1927. There is also added to the list a number of species more recently described by C. P. Alexander, J. R. Malloch, C. H. Curran, and A. L. Melander, the names of the collectors being given in connection with the species described. The following list contains about 170 species.

TRICHOCERIDE

Trichocera Meigen

T. sp. Nain, Aug. 18, Kangalasiornik, Sept. 15 (Bryant).

\section{TIPULIDAE}

Dicranomyia Stephen

D. halterata O.S. Battle Harbor, July 11.

Dactylolabis Osten Sacken

D. rhicnoptiloides Alex.

Limnophila (Dactylolabis) rhicnoptiloides Alex. Rept. Can. Arctic Exped. 1913-18, Crane-flies, p. 6 c. 1919.

The type was taken at Bernard Harbor, N. W. Ter- 
ritories. It was later recorded from Ellesmereland by Dr. Alexander, collected on the Second "Fram" Expedition.

A specimen of this species was taken at Joksut, an inlet just south of Cape Chidley, Aug. 17, 1927, by O. L. Austin, Jr. Specimen in the Museum of Comparative Zoölogy.

\section{Chionea Dalman}

C. waughi Curran. Can. Ent. vol. .57, p. 24, 1925. Cabot Lake, Sept-Oct. and Voisey's Bay, Dec. 5, 1921 (F. W. Waugh).

\section{Limnophila Macquart}

L. subunica Alex. Rama, 1899 (Stecker).

\section{Tricyphona Zetterstedt}

T. hyperborea O. S. "Labrador." (Osten Sacken).

'T. inconstans O. S. Cape Charles, July 28 (Allen).

\section{Dolichopeza Curtis}

D. americana Needh. Rigolet, July 16 (Allen). .

\section{Prionocera Loew}

P. electa Alex. Can. Ent., vol. 59, p. 188, 1927. Hopedale, July 1, 1923 (W. W. Perrett).

\section{Tipula Linnæus}

T. angustipennis Loew. Hopedale and Caribou Isl. (Packard). Rama (Sornborger).

T. aperta Alex. Can. Ent., vol. 40, p. 62, 1918. (T. imperfecta Alex. 1915 non Brunetti. 1913). "Labrador" (Packard).

T. canadensis Loew, Hopedale, July 31, 1922, June 21, 1924. (W. W. Perrett).

T. entomophthoræ Alex. Can. Ent., vol. 60, p. 99, 1928 Hopedale, June 19-27, 1925 (W. W. Perrett).

T. grenfelli Alex. Can. Ent., vol. 60, p. 96, 1928. Hopedale, July 7, 1923 (W. W. Perrett). 
T. labradorica Alex. Insec. Inscit., vol. 3, p. 128, 1915. (T. tessellata Loew 1863 non T. tessellata de Villers 1789 Battle Harbor July 11, Great Caribou Isl., July 14 (Allen).

T. macrolabis Loew. Rama, Aug. 24, 1882 (Sornborger Hopedale, June 19-Aug. 4, (W. W. Perrett).

T. nebulipennis Alex. Battle Harbor, Aug. 1, 1912 (G. P. Engelhart).

T. packardi Alex. Can. Ent., vol. 60, p. 99, 1928. Hopedale, Aug. 24, 1924 (W. W. Perrett).

T. perretti Alex. Can. Ent., vol. 60, p. 98, 1928. Hopedale, July 17, 1923. (W. W. Perrett).

T. productella Alex. Can. Ent., vol. 60, p. 100, 1928. Hopedale, Sept. 13, 1923 (W.W. Perrett).

T. septentrionalis Loew. "Labrador" (Schneider) Hopedale, June 18-July 5 (W.W. Perrett).

T. serta Loew (T. albonotata Doane). Hopedale, June 19 to Aug. 4 (S.S. Perrett).

T. subserta Alex. Can. Ent. vol. 60, p. 97, 1928. Hopedale (Packard). Rama 1894 (Sornborger). Hopedale July 18-25 (W.W. Perrett).

\section{CULICIDÆE}

Aedes Meigen

A. punctor (Kirby). A. provocans Walk. St. Lewis Inlet, Rigolet and Cape Charles July 12 to 28 (Allen).

\section{CHIRONOMIDE}

Culicoides Latreille

C. sp. "Labrador" (Packard).

Tanypus Meigen

T. monilis Linn. Great Caribou Isl., July 26 (Allen).

T. sp. Hopedale (Packard). 


\section{Chironomus Meigen}

C. annularis De G. Hopedale (Packard), Great Caribou Isl., July 27 (Allen).

C. cristatus Fab. Hopedale (Packard), Great Caribou Isl., July 27 (Allen).

C. riparius Meig. Hopedale (Packard).

C. brunneipes Zett.? Hopedale (Packard) Cape Charles, July July 29 (Allen).

\section{CECIDOMYIID $A$}

\section{Rhaỏdophaga Westwood}

R. sp. Hopedale, (Packard).

Packard in his Labrador Coast, p. 390, records Micromyia leucorum with the following note: "Prof. C. W. Woodworth writes me that on examining the collection of Diptera which I made in Labrador, and which is now in the Cambridge Museum, he detected the rare European Cecidomyid, Micromyia leucorum, belonging to a genus hitherto unrecorded for North America."

I have examined the specimen referred to and thus labeled in the Museum of Comparative Zoölogy, and find that it is a Chironomid.

\section{MYCETOPHILIDAE}

Gnoriste Meigen

G. megarrhina Osten Sacken, Nain, Aug. 18, (Bryant).

Mycomya Rondani

M. maxima Johannsen, Nain Aug. 18 (Bryant). The type locality "Maine," should read Nain.

\section{Allocadia Winnertz}

A. sp. Nain Aug. 18 (Bryant).

\section{Neosciara Petty}

N. sp. St. Lewis Inlet, July 12 (Allen). 


\section{BIBIONIDEE}

\section{Bibio Geoffroy}

B. inæqualis Loew $\delta$ \& Hopedale (Packard). Rama 1899, (Stecker and Sornborger). Cape Charles July 29 and 30 (Allen).

Bibio bryanti sp. nov.

o Head, antennæ and palpi black, pile of the eyes long and brown. Thorax black, shining with long black pile. Abdomen black, whitish pile. Legs reddish, with yellowish hairs, coxæ and knees black, the inner spur of the front tibiæ about one-half the length of the outer one, the posterior tibiæ and metatarsi enlarged, the latter about as long as the second and third joint combined. Wings hyaline, the costa, stigma and first and second veins brown. Halteres dark brown. Length $8 \mathrm{~mm}$. Nain, Aug. 18 (Bryant). Rama, Aug. 24 (Sornborger). Holotype and paratypes in the $\mathrm{Mu}$ seum of Comparative Zoölogy.

It resembles $B$. variabilis Loew, but the lighter colored femora and enlarged metatarsi readily separates it. From $B$. fumipennis Walk, it is distinguished by its light colored wings and veins and the noticeably thicker tibiæ and metatarsi.

Bibio labradorensis sp. nov.

of Head, antennæ and palpi black, the pile on the eyes brown. Thorax black, shining, pile yellowish. Abdomen black pile yellow. Femora black, tibiæ and tarsi yellow, the latter very short, the posterior tibiæ as large as the femora and posterior metatarsi about one-half the diameter of the tibia, the second joint of the tarsus also noticeably enlarged. Wings hyaline, the anterior veins and stigma light yellow. Halteres dark brown. Length $5.5 \mathrm{~mm}$. Two specimens. Nain, Aug. 18, 1908 (Bryant).

This species resembles a small $B$. variabilis Loew, but differs in having thickened metatarsi and obsolete stigma. Holotype and paratype in the Musem of Comparative Zoölogy. 
SCATOPIDAE

Aspistes Meigen

A. analis Kirby. Hopedale (Parkard).

\section{SIMULIID $\approx E$}

Simulium Latreille

S. venustum Say. Cape Charles, July 29 (Allen).

S. (Wilhelmia) vittatum Zett. Fort Chimo (Turner). Hawk's Harbor, July 20, '08. (Peary's North Pole Expd.)

\section{Prosimulium Roubaud}

P. hirtipes Fries. Rigolet and Cape Charles, July 18 and 28 (Allen). Nain, Aug. 18 (Bryant). Hawk's Harbor, July 20, '08 (Peary's North Pole Expd.)

\section{STRATIOMYIDAE}

Beris Latreille

B. annulifera var. brunnipes Johns. Psyche, vol. 33, P. 109, 1926. Parroquet Isl., July 21, 1881 (Henshaw). Strait of Belle Isle (Packard.)

\section{TABANID $A$}

Chrysops Meigen

C. excitans Walk. St. Lewis Inlet, July 12 (Allen).

C. mitis O.S. St. Lewis Inlet, Great Caribou Isl. and Cape Charles, July 12 to Aug. 29 (Allen).

C. sordida O.S. Great Caribou Isl. July 29 (Allen).

Tabanus Linnæus

T. affinis Kirby, Great Caribou Isl., 27 (Allen).

T. astutus O.S. Great Caribou Isl. and Cape Charles, July 27-30 (Allen). 
T. flavipes Wied. St. Lewis Inlet and Cape Charles, July 12 and 21. (Allen) Kangalasiornik Bay, Sept. 5 (Bryant).

T. illotus O.S., St. Lewis Inlet, July 12 (Allen).

T. septentrionalis Loew. Great Caribou Isl. and Cape Charles July 27-30 (Allen). Nain Aug. 18 (Bryant).

T. zonalis Kirby. Lewis Inlet, and Cape Charles, July 12 and 28 (Allen).

T. labradorensis Enderlein. (Tyloslypia labradorensis Ender. mitt. Zoöl. Mus. Berlin, xi, 363, 1925). "Labrador." (Enderlein).

T. cristatus Curran. Can. Ent., vol. 59, p. 81, 1927. "Labrador" (Curran).

RHAGIONIDAE

Symphoromyia Frauenfeld

S. montana Aldr. Ungava Bay (Turner).

ASILID 25

Asilus Linnæus

A. nitidifacies Hine. Hopedale, Aug. 19, 1923 (W. W. Perrett).

\section{DOLICHOPODIDAE}

Dolichopus Latreille

D. annulipes Zett. (D. stenhammeri Zett). Sloop Harbor, July 19, Hopedale and Caribou Isl. (Packard). Nain, Aug. 18 (Bryant). Dr. Aldrich agrees with Becker that annulipes is not preoccupied, c.f. Proc. U.S. Nat. Mus., vol. 61, art. 25, p. 2, 1922.

D. boreus Van D., Cole and Aldr. Ungava Bay, July 22-28 (Turner).

D. brevipennis Meig. Nain Aug. 18 (Bryant).

D. bryanti Van D., Cole and Aldr. Hopedale and Strawberry Harbor (Packard). Ungava Bay, July 29 (Turner) Great Caribou Isl. July 27 and Nain, Aug. 18 (Bryant).

D. dasypodus Coq. Hopedale and Caribou Isl. (Packard). Nain, Aug. 18 (Bryant). 
D. delicatus Aldr. Ungava Bay and Fort Chimo (Turner).

D. groenlandica Zett. Ungava Bay, July 29 (Turner). Nain, Aug. 18 (Bryant).

D. packardi Van D., Cole and Aldr. Nain, Aug. 18 (Bryant).

D. latronis Van D., Cole and Aldr. Hopedale (Packard).

Hydrophorus Fallen

H. chrysologus Walk. Strait of Belle Isle (Packard).

Scellus Loew

S. filifer Loew. Nain, Aug. 18 (Bryant).

The nine specimens, all females, are a little smaller than specimens from Farwell Creek, South Saskatchewan (Mrs. Armstrong), and High River, Alberta (Owen Bryant).

Rhaphium Meigen

R. sp. Hopedale (Packard), Caribou Isl. (Packard). "Both specimens are females that I cannot determine." (M. C. Van Duzee).

EMPIDIDAE

Bicellaria Macquart

B. uvens Mel. (Genera Ins., 74 1927) Fort Chimo, Ungava Bay (Turner).

Iteaphila Zetterstedt

I. curva Curr. Can. Ent., vol. 57, p. 24, 1925. Nain, June 1522 (F. W. Waugh).

Hilara Meigen

H. unicolor Loew. Caribou Isl. (Packard) Nain, Aug. 18 (Bryant).

\section{Empis Linnæus}

E. lævigata Loew. Battle Harbor, July 11; Cape Charles, July 28 (Allen). Caribou Isl., Indian Harbor and Strawberry Harbor (Packard).

E. obesa Loew Nain, Aug. 18 (Bryant). 
Rhamphomyia Meigen

R. incompleta Loew. Nain, Aug. 18 (Bryant).

R. setosa Coq. Great Caribou Isl., July 27 (Allen). Nain, Aug. 18 (Bryant). Strawberry Harbor (Packard).

Clinocera Meigen

C. (Hydrodromia) longifurca Mel. (Genera Ins., 231, 1927) Nain, Aug. 18 (Bryant).

C. (Hydrodromia) conjunta Loew. Great Caribou Isl., July 27 (Allen). Nain, Aug. 18 (Bryant).

Chelifera Macquart

C. (Metachela) albipes Walk. Fort Chimo, Aug. 11 (Turner).

Tachypeza Meigen

T. winthemi Zett. Great Caribou Isl., Aug. 11.

Platypalpus Macquart

P. arcticus Mel. (Genera Ins., 344, 1927). Fort Chimo, Ungava Bay (Turner).

P. melanogaster Mel. (Genera Ins., 323, 1927). Ungava Bay (Turner).

P. prorsus Mel. (Genera Ins., 328 1928). Ungava Bay (Turner).

P. satyriacus Mel. (Genera Ins., 327, 1927) Ungava Bay, (Turner).

P. xanthopus Mel. (P. gilvipes Coq. not Meig.) Genera Ins., 367, 1927. Great Caribou Isl., July 14 and 27 (Allen).

\section{PHORIDFE}

Megaselida Rondani

M. rufipes Meig. Nain, Aug. 18 (Bryant). 


\section{SYRPHIDE}

\section{Pyrophæna Schiner}

P. granditarsus Forster "Labrador" (Henshaw).

Platychirus St. Fargeau and Serville.

P. albimanus Fabr. Great Caribou Isl., July 21 (Allen). Nain, Aug. 18 (Bryant).

P. hyperboreus Stæg. Strawberry Harbor (Packard). Nain, Aug. 18 (Bryant).

P. peltatus Meig. dark var. Two females, Nain, Aug. 18 (Bryant).

P. quadratus Say. Strait of Belle Isle (Packard).

\section{Melanostoma Schiner}

M. mellinum Linn. Strawberry Harbor (Packard).

M. obscurum Say. Strawberry Harbor (Packard).

\section{Syrphus Fabricius}

S. pallifrons Curran Kansas Univ. Sci. Bull., vol. 15, p. 172, 1924. Nain, Aug. 18 (Bryant).

S. perplexus Osburn. Kangalasiornik Bay, Sept. 5, (Bryant.

\section{Epistrophe Walker}

E. contumax O. S. Indian Harbor (Packard). Rama (Stecker and Sornborger). Great Caribou Isl., July 14 (Allen).

E. diversipes Macq. Indian Harbor, Strawberry Harbor and Caribou Isl. (Packard).

E. geniculatus Macq. Nain, Aug. 18 (Bryant).

E. insoletus Osburn St. Lewis Inlet, July 12 (Allen).

Sphæropheria St. Fargeau et Serville

S. strigata Stæger. Strawberry Harbor (Packard). Great Caribou Isl, July 14 (Allen). Nain Aug. 18 (Bryant). 


\section{Volucella Geoffroy}

V. bombylans arctica Johnson.

V. facialis form arctica Johnson Psyche, vol. 23, p. 163, 1916.

$V$. bombylans arctica Johnson. Psyche, vol. 32, p. 116, 1925.

Rama, 1899 (Stecker and Sornborger), Nain (Sornborger), Nain, Aug. 18, 1908 (Bryant).

This circumpolar group of Volucella that live in the nests of various species of Bombus, form an interesting but difficult group to classify. Recognizing but one species with numerous subspecies seems to be the most satisfactory method of treating them.

Eristalis Latreille

E. arbustorum Linn. Nain, Aug. 18 (Bryant) Battle Harbor (Englehardt).

E. bastardii Macq. "Labrador" (Williston).

\section{Helophilus Meigen}

H. borealis Stæger. Rama (Stecker and Sornborger), Nain, Aug. 18 (Bryant).

H. groenlandicus O. Fab. Indian Harbor (Packard), Nain, Aug. 18 (Bryant).

H. latifrons Loew. Nain, Aug. 18 (Bryant).

\section{OESTRIDAE}

Cephenomyia trompe Linn?

The species that infests the Barren-ground Caribou (Rangifer articus) is probably the same that frequents the reindeer and is circumboreal in distribution. The adult fly has not been taken. Dr. Grenfell informs me that in all the heads of the caribou that he has examined he found parasitic larvæ usually just below the ethmoid. See Insects of Labrador, in appendix to "Labrador the Country and the People" by Wilfred T. Grenfell, 1909. 


\section{Oedemagena tarandi Linn?}

This is also probably the same species that infests the reindeer. The larvæ are subcutaneous, forming large lumps on the back, from which they escape when full grown through holes in the skin. Dr. Grenfell says that he has seen a skin so perforated that it was practically impossible to cut from it a pair of moccasins. The fly has not been taken in Labrador.

\section{TACHINID $F$}

Linnæmyia R.-Desvoidy

L. varia Curran. Hopedale, June 12-July 18 (Rev. Perrett).

Fabriciella Bezzi

F. orientalis Town. (? Tachina florum Walk.) Cape Charles, July 3 (Allen).

F. montana Town. Nain (Sornborger).

SARCOPHAGIDAE

Pachyophthalmus Brauer and Bergenstamm

P. distortus Allen (Proc. U.S. Nat. Mus., vol. 68, art. 9, p. 15, 1926). Hamilton Riv., Muskrat Falls, July 12-19, 1919 (S. E. Arthur).

\section{CALLIPHORIDAE}

\section{Protophormia Townsend}

P. terrænovæ R.-Desv. "Labrador” (Sornborger).

\section{Cynomyia R.-Desvoidy}

C. mortuorum Linn. Nain, Aug. 18 (Bryant).

C. cadaverina R.-Desv. Square, Isl. (Packard). Rama and Hebron (Sornborger). Kangalasiornik Bay, Aug. 28 (Bryant). 


\section{Steringomyia Pokorny}

S. flavipalpis Macq. Indian Harbor (Packard).

Boreellus Aldrich and Shannon

B. atriceps (Zetterstedt).

Sarcophaga atriceps Zett. Dipt. Scand., vol. 4, 1311, 31 1843.

Melinda atriceps Hend. Wien. Ent. Zeitz., xx 33, 1901. Onesia atriceps Zett. Katal. Pal. Dipt., III, 550, 1907 Phormia cærulea Mall., Rept. Can. Arctic Expd., vol. 3, pt. c, p. 61 c, 1919.

Boreellus aristatus Aldr. \& Shan., Insec. Inscit., xi, 107, 1923.

Boreellus atriceps Shan., Proc. Ent. Soc. Wash., vol. 28, p. 128, 1926.

Mallochomyia johanseni Town. Insec. Inscit., Vol. 14, p. 25, 1926. Gready Harbor, Aug. 8 (Bryant).

Calliphora R.-Desvoidy

C. vomitoria Linn. Indian Harbor (Packard).

$$
\text { MusCID } Æ
$$

Musca Linnæus

M. domestica Linn. near Killinek, Sept. 5 (Bryant).

\section{Myospila Rondani}

M. meditabunda Fab. Great Caribou Isl., July 27 (Allen). ANTHOMYIIDA

\section{Phaonia R.-Desvoidy}

P. errans Meig. Square Isl., July (Packard).

P. monticola Mall. Rama and Indian Harbor (Packard). Nain, Aug. 18 (Bryant).

P. serva Meig. Strait of Belle Isle. (Packard). Rigolet, July 5 (Allen). 
Trichopticus Rondani

T. innocuus Zett. Strawberry Harbor (Packard). Great Caribou Isl., July 27 (Allen). Nain, Aug. 18 (Bryant).

T. septentrionalis Stein., Nain. Aug. 18 (Bryant).

T. spiniger Stein. Nain, Aug. 18 (Bryant). Caribou Isl. Packard).

\section{Rhynchotrichops Schnabl}

R. subrostrata Zett. .Battle Harbor, July 11 (Packard). Great Caribou Isl., July 14 (Allen).

\section{Pogonomyia Rondani}

P. quadrisetosa Mall. Can. Arctic. Expd., vol. 3, pt. c. p. 66c, 1919. Nain.

\section{Helina R.-Desvoidy}

H. fulvisquama Zett. (H. tuberculata Mall., Can., Ent., Vol. 51, p. 277, 1919, not Mall., Trans. Amer. Ent. Soc., vol. 46, p. 138, 1920). Rigolet, July 18 (Allen).

H. duplicata Meig. Caribou Isl. (Packard).

\section{Spilaria Schnabl.}

S. lucorum Fall. Nain, Aug. 18 (Bryant).

S. marmorata Zett. Caribou Isl. and Straits of Belle Isle (Packard).

$$
\text { Hydrotæa R.-Desvoidy }
$$

H. cristata Mall. Indian Harbor (Packard).

Fannia R. Desvoidy

F. scalaris Fab. Caribou Isl. (Packard).

Spilogona Schnabl and Dziedzicki

S. alticola (Mall.) Trans. Amer. Ent. Soc., vol. 46, p. 153, 1920. Nain, Aug. 18 (Bryant).

S. sp. Nain, Cape Charles and Caribou Isl. 
Lispocephala Pokorny

L. erythrocera Desv. Caribou Isl. and Strawberry Harbor (Packard).

Conosia Meigen

C. humilis Meig. Cape Charles, July 29 (Allen). Caribou Isl. (Packard).

\section{Prosalpia Pokorny}

P. angustitarsis Mall. Nain, Aug. 18 (Bryant).

P. silvestris Fab. Straits of Belle Isl., Indian Harbor (Packard).

\section{Hylemyia R. Desvoidy}

H. brassicæ Bouche. Nain, Aug. 18. Killinek, Sept. 5 (Bryant).

H. cilicrura Rond. Caribou Isl. (Packard).

H. antiqua Meig. Hopedale (Packard).

\section{Egle R.-Desvoidy}

E. mystacea Coq. "Labrador (Malloch). Nain, Aug. 18 (Bryant).

E. muscaria Meig. Strawberry Harbor and Hopedale (Packard).

Paregle Schnabl and Dziedzicki

P. radicum Linn. Nain, Aug. 18 (Bryant).

Pegomyia R.-Desvoidy

P. fuscofasciata Mall. Nain, Aug. 18, (Bryant). Caribou Isl. and Strawberry Harbor (Packard).

P. labradorensis Mall. (Trans. Amer. Ent. Soc., vol. 46, p. 176, 1920 Nain, Aug. 18. Kangolasiornik, Sept.(Bryant). Indian Harbor (Packard). 
Fucellia R.-Desvoidy

F. maritima Haliday, Great Caribou Isl. July 14 (Allen). Caribou Isl. (Packard).

\section{SCATOPHAGIDEE}

\section{Scatophaga Meigen}

S. stercoraria Linn. Indian Harbor and Caribou Isl. (Packard). Ragged Isl. July 23.

S. islandica Becker. Hopedale, Indian Harbor, Caribou Isl. and Strawberry Harbor (Packard). Hebron, Aug. 28. Rama, Aug. 24, 1897 (Sornborger). Great Caribou Isl., July 14, Gready Harbor Aug. 8, Nain, Aug. 18 (Bryant).

S. furcata Say. Ungava Bay (Turner). Nain, Rama, Hopedale and Indian Harbor (Packard). Great Caribou Isl. (Allen). Gready Harbor, Aug. 8 (Bryant).

S. estotilandia Rondani

Scatina estotilandia Rond. Archiv. Canestrini, III, fasc. I, p. 35, 1863. Archiv. Zoöl. Modena, Vol. 3, 35, 1863. Labrador. Osten Sacken, Catl. Dipt. N. Amer., 1878, p. 174, says: "Mr. Rondani in the same place mentions Scatophaga diadema Wied. (Montevideo) as having been received from Labrador." Hendel (Wien. Ent. Zeit., vol. 29, p. 116, 1910) in a footnote under Thyreophorella diadema (Scatophaga diadema Wied.) says :"The insect that Rondani determined as Scatina diadema Wied., is doubtless a Scopeuma [Scatophaga] but not likely to be the above species." [S. diadema].

Trichopalpus Rondani

T. sp. Caribou Isl. (Packard); Nain, Aug. 18 (Bryant).

Pogonota Zetterstedt

P. kincaidii Coq. Nain Aug. 18 (Bryant).

Microprosopa Becker

M. hæmorrhoidalis Meig. Nain, Aug. 18 (Bryant). 
Eugenacephala Johnson

E. ruficeps Curran (1925). Natashquan June-July, 1921. (F. W. Waugh).

\section{Acanthocnema Becker}

A. albibarba Loew. Nain, Aug. 18, Bryant. The type from the White Mts., N. H., is teneral.

HELOMYZID $A$ E

Suillia R.-Desvoidy

S. zetterstedtii Loew. Ungava Bay (Turner).

Allophyla Loew

A. lævis Loew Caribou Isl. (Packard).

Oecothea Haliday

o. fenestralis Fall. Indian Harbor (Packard).

Scoliocentra Loew

S. fraterna Loew. Ungava Bay (Turner).

Neoleria Malloch

N. ruficauda Zett. (Leria crassipes Loew) Ungava Bay (Turner). Gready Harbor, Aug. 8. Nain, Aug. 18 (Bryant).

BORBORIDAE

Borborus Meigen

B. arcticus Mall. Fort Chimo, Ungava Bay (Turner).

SCIOMYZIDAE

Dictya Meigen

D. umbrarum Linn. Great Caribou Isl., July 29 (Allen). 
SAPROMYZIDE

Minettia R. Desvoidy

M. lupulina Fab. Strawberry Harbor, Hopedale (Packard).

PALLOPTERIDAE

Palloptera Fallen

P. jucunda Loew. Parroquet Isl., July 27, 1881 (S. Henshaw).

PIOPHILIDAE

Piophila Fallen

P. casei Linn. Indian Harbor and Hopedale (Packard).

EPHYDRIDAE

Scatella R.-Desvoidy

S. stagnalis Fall. Nain, Aug. 18 (Bryant). Hopedale, Strawberry Harbor (Packard).

CHLOROPID $\approx$

Chlorops Meigen

C. melanocera Loew, Nain, Aug. 18. Kangalasiornik Bay, Sept. 5, (Bryant). 

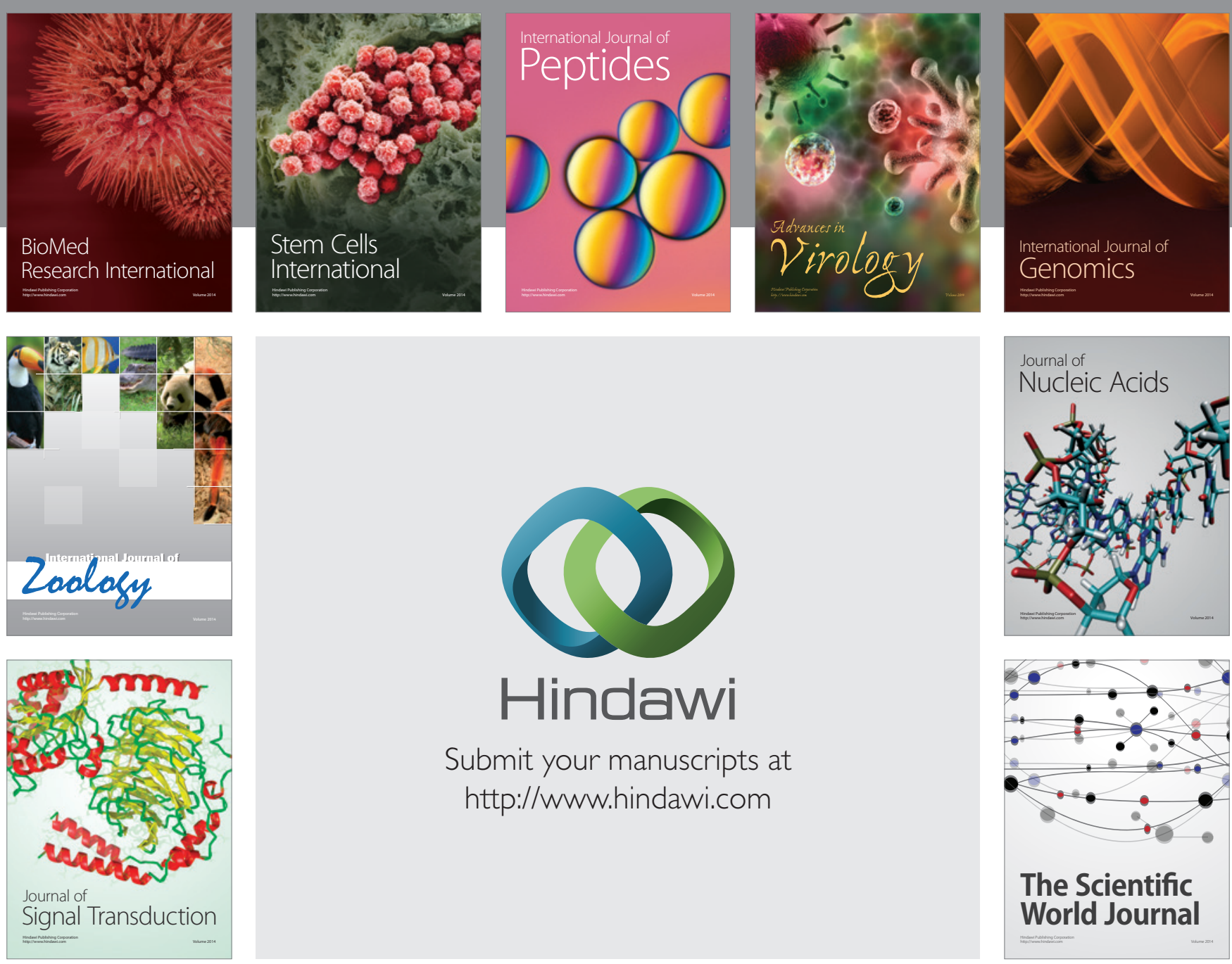

Submit your manuscripts at

http://www.hindawi.com
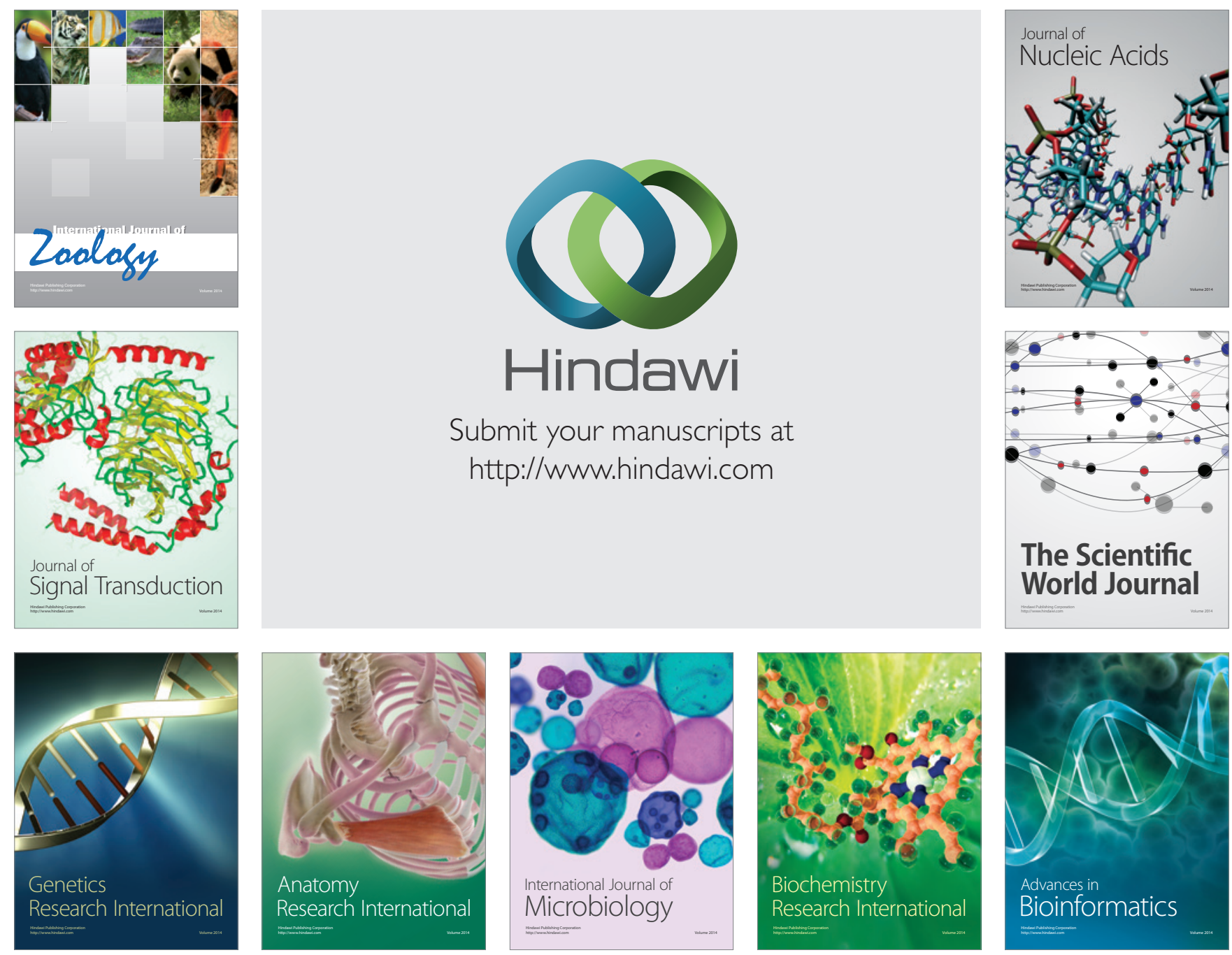

The Scientific World Journal
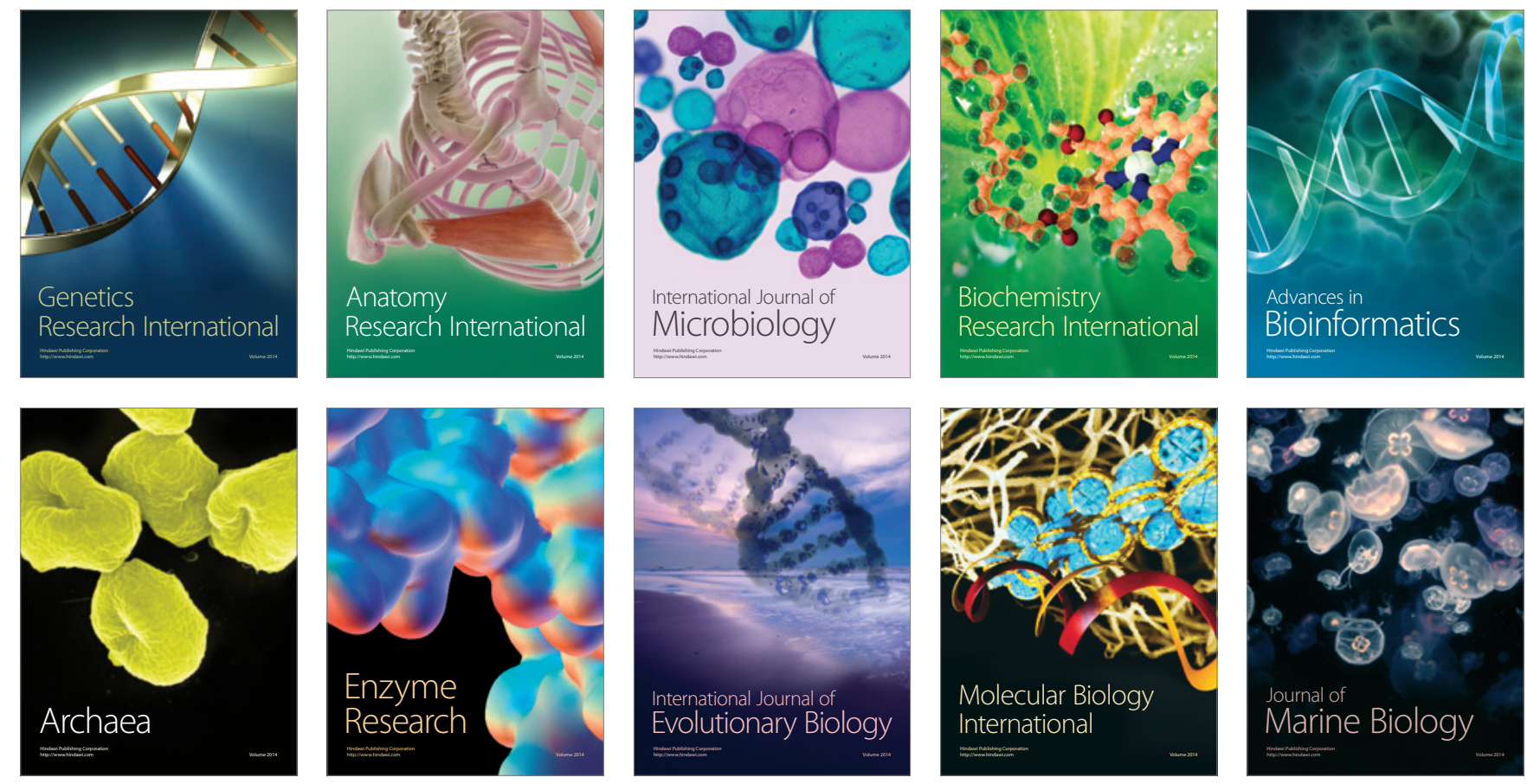